\title{
Influence of Polydatin on the Blood Lipid of Rat with Hyperlipemia
}

\author{
Ouyang Enhong ${ }^{1}$, Junhua Liu ${ }^{2}$, Hailiang $\mathrm{Wei}^{1}$, Gengxi $\mathrm{Li}^{1}$ \\ ${ }^{1}$ Department of pharmacy, Shaoyang University, Shaoyang Hunan, 422000, China; \\ ${ }^{2}$ Department of Pharmacology, Affiliated Hospital of Shaoyang University, Shaoyang Hunan, 422000, \\ China
}

Keywords: Polydatin, Atherosclerosis, Hyperlipemia.

\begin{abstract}
Objective: Discuss the regulating effect of polydatin on the blood lipid of rat with with hyperlipemia. Method: rats were fed with the high-fat feeding method; the early atherosclerosis (AS) model of rat was established; after successful modeling, rats were given by gavage high-dose, medium-dose and low-dose of polydatin $\left(160,80,40 \mathrm{mg} \cdot \mathrm{kg}^{-1} \cdot \mathrm{d}^{-1}\right) ; 8$ weeks later, rats' TC, TG, HDL-C, LDL-C, TNF- $\alpha$, NO and iNOS were measured. Result: the group with high-dose, medium-dose and low-dose of polydatin can significantly lower the serum TC, TG, LDL-C, TNF- $\alpha$, NO level and iNOS activity and enhance the HDL-C. The difference is of statistical significance ( $\mathrm{P}$ $<0.05)$. Conclusion: Polydatin can regulate blood lipids and alleviate the occurrence and development of atherosclerosis. This may be related to its regulation of the formation and expression of NO and nitric oxide synthase.
\end{abstract}

\section{Introduction}

The incidence of cardiovascular and cerebrovascular diseases (CCVD) is gradually on the rise in our country and atherosclerosis (AS) is the direct cause for CCVD, so prevention and treatment of AS are of great importance for the prevention and treatment of such diseases. Polydatin is an important active monomer ingredient extracted from Polygonum cuspidatum, a traditional Chinese medicine. Modern pharmacological researches show that polydatin has many biological activities. Through the experimental study on the intervention effect of polydatin on early AS of rats with hyperlipemia and the preliminary study on its intervention effect, this paper can offer a new theoretical basis for the development of polydatin as an antiatherosclerotic drug and offer a new experimental basis for the application of traditional Chinese medicine in anti atherosclerosis.

\section{Materials}

\section{Drugs and regents}

Polydatin (batch No.: 150731; supplied by Shanghai Chunsheng Biotechnology Co., Ltd.; content > 98\%; relative molecular mass: 1 806); Simvastatin (batch No.: 150292; specification: 20 mg; Hangzhou MSD Pharmaceutical Co., Ltd.); TC kit (batch No.: 20150907); TG kit (batch No.: 20150728); HDL-C kit (batch No.: 20150910); LDL-C kit (batch No.: 20150729). All of the kits above were supplied by Sigma Company. TNF- $\alpha$ ELISA kit (R\&D Corp. of America); NO kit measured through nitric acid reductase method (batch No.: 20151117; Nanjing Jiancheng Bioengineering Institute); iNOS kit (batch No.: 20150112; Nanjing Jiancheng Bioengineering Institute).

\section{Instruments}

Animals digital display thermostat water bath (Guohua Electric Appliance Co., Ltd.); BS224s electronic scales (Beijing Sartorius Instrument System Co., Ltd.); BIO-RAD Model680 microplate reader (Bio-Rad of America); Mikro22R high-speed refrigerated centrifuge (Hettich of German). 


\section{Animals}

SD rats, male, weight: 200-250g; supplied by Jinan Jinfeng Experimental Animal Breeding Co., Ltd.; license No.: SCXK(Lu)20150006.

\section{Fodder}

Formula of high-fat fodder: $3 \%$ cholesterol; $0.5 \%$ sodium cholate; $10 \%$ lard; $0.2 \%$ propylthiouracil; 5\% white sugar; $81.3 \%$ basal feed. All of them were offered by Zhejiang Experimental Animal Center.

\section{Method}

\section{Modeling and grouping of rats with hyperlipemia}

SD rats were randomly divided into 6 groups with 10 rats in each group. Normal diets were given to the normal control group and high-fat diets were given to other groups. After they were fed for 4 weeks, blood was drawn from their orbits to measure the blood lipid; then, the high-fat groups were randomly divided into 5 groups based on their blood lipid levels: (1) model control group: ig distilled water. (2) positive drug Simvastatin group: ig $5 \mathrm{mg} \cdot \mathrm{kg}^{-1} \cdot \mathrm{d}^{-1}(3)$ high-dose polydatin group: ig 160 $\mathrm{mg} \cdot \mathrm{kg}^{-1} \cdot \mathrm{d}^{-1}$. (4) medium-dose polydatin group: ig $80 \mathrm{mg} \cdot \mathrm{kg}^{-1} \cdot \mathrm{d}^{-1}$.5) low-dose polydatin group: ig $40 \mathrm{mg} \cdot \mathrm{kg}^{-1} \cdot \mathrm{d}^{-1}$. From the $5^{\text {th }}$ week, each group was fed with drugs (or distilled water) for 8 weeks; meanwhile, the normal control group was given normal diets while other groups were always fed with high-fat diets.

\section{Body weight determination}

The rats were weighed before modeling, in the $4^{\text {th }}, 8^{\text {th }}$ and $12^{\text {th }}$ weeks after modeling; changes in their weights were recorded.

\section{Measurement of blood lipid}

After the last administration, blood was collected from their orbital veins; after 30 min of standing, serum was separated centrifugally; then the TC, TG, LDL-C and HDL-C concentrations of serum were detected according to the instructions on the kit.

\section{Data processing}

All experimental materials were expressed with $\bar{x} \pm$ s. The ANOVA analysis of variance was adopted for comparison among groups. When $\mathrm{P}<0.05$, it means that the difference is significant.

\section{Result}

\section{Changes in rat weights of each group}

The weights of rats in the normal control group gradually increase with time extension; the weights of rats in the model group increase slowly and are significantly less than those in the blank control group ( $\mathrm{P}<0.01)$. The weights of rats in the Simvastatin group and high-dose polydatin group slightly increase, compared with the model group, but there is no significant difference. For the result, see Table 1. 
Table 1. Changes in rats' weights before modeling and in the 4th, 8th and 12th weeks after modeling (n=10, $\bar{x}_{ \pm s)}$

\begin{tabular}{cccccc}
\hline \multirow{2}{*}{ Group } & \multirow{2}{*}{$\begin{array}{c}\text { Dose } \\
(\mathrm{mg} \cdot \mathrm{kg}-1)\end{array}$} & \multicolumn{4}{c}{ Weight/g } \\
\cline { 3 - 6 } & & Before administration & 4th week & 8th week & 12 th week \\
\hline Normal control group & & $212.5 \pm 13.0$ & $290.6 \pm 25.3$ & $314.3 \pm 18.0$ & $386.2 \pm 16.5$ \\
Model group & & $201.8 \pm 11.0$ & $217.9 \pm 16.6$ & $225.4 \pm 15.0$ & $230.2 \pm 10.4 *$ \\
& & & $*$ & $*$ & $241.8 \pm 20.6 *$ \\
Simvastatin group & 160 & $199.7 \pm 8.9$ & $217.2 \pm 18.4$ & $229.6 \pm 14.6$ & $*$ \\
High-dose polydatin group & 80 & $206.7 \pm 13.0$ & $220,3 \pm 15.6$ & $228.0 \pm 19.0$ & $241.9 \pm 20.8$ \\
Medium-dose polydatin group & 40 & $210.0 \pm 11.6$ & $216.2 \pm 15.7$ & $224.0 \pm 7.8$ & $228.2 \pm 15.7$ \\
Low-dose polydatin group & & $204.6 \pm 11.8$ & $225.6 \pm 16.2$ & $228.1 \pm 8.9$ & $224.5 \pm 15.9$ \\
\hline
\end{tabular}

*Compared with the normal control group: $\mathrm{P}<0.01$;** compared with the model control group, $\mathrm{P}<0.05$.

\section{Influence of polydatin on the blood lipid of rat}

Compared with the blank control group, the TC, TG and LDL-C contents in serum of rats in the model group rise significantly ( $\mathrm{P}<0.01$ ); the HDL-C content decreases significantly $(\mathrm{P}<0.01)$; it means the successful hyperlipemia modeling. Compared with the model control group, the high-dose polydatin group can significantly lower the TC(P $<0.01)$, TG(P $<0.01)$ and LDL-C $(\mathrm{P}<0.01)$, and significantly increase the HDL-C $(\mathrm{P}<0.05)$; besides, the functions of all doses of polydatin show a certain dose dependence. The result is shown in Table 2.

Table 2. Influence of polydatin on the TC, TG, LDL and HDL in the serum of rat with hyperlipemia ( $\left.\mathrm{n}=10, \bar{x}_{ \pm s}\right)$

\begin{tabular}{|c|c|c|c|c|c|c|}
\hline Group & Dose & (mg·kg-1) & $\begin{array}{c}\mathrm{TG} \\
(\mathrm{mmol} \cdot \mathrm{L}-1)\end{array}$ & $\begin{array}{c}\mathrm{TC} \\
(\mathrm{mmol} \cdot \mathrm{L}-1)\end{array}$ & $\begin{array}{c}\text { LDL } \\
(\mathrm{mmol} \cdot \mathrm{L}-1)\end{array}$ & $\begin{array}{c}\text { HDL } \\
(\mathrm{mmol} \cdot \mathrm{L}-1)\end{array}$ \\
\hline Normal control group & & & $\begin{array}{c}0.542 \pm 0.100 * \\
*\end{array}$ & $\begin{array}{c}1.542 \pm 0.100 * \\
*\end{array}$ & $\begin{array}{c}0.554 \pm 0.091 * \\
*\end{array}$ & $\begin{array}{c}1.403 \pm 0.144 * \\
*\end{array}$ \\
\hline Model group & & & $1.940 \pm 0.086$ & $2.782 \pm 0.267$ & $1.432 \pm 0.113$ & $0.886 \pm 0.056$ \\
\hline Simvastatin group & & 5 & $\begin{array}{c}1.814 \pm 0.076 * \\
*\end{array}$ & $2.492 \pm 0.321 *$ & $\begin{array}{c}0.803 \pm 0.116 * \\
*\end{array}$ & $\begin{array}{c}1.329 \pm 0.167 * \\
*\end{array}$ \\
\hline High-dose polydatin group & 160 & & $\begin{array}{l}1.753 \pm 0.078 * \\
*\end{array}$ & $\begin{array}{l}2.256 \pm 0.099 * \\
*\end{array}$ & $\begin{array}{l}0.874 \pm 0.046 * \\
*\end{array}$ & $\begin{array}{l}1.298 \pm 0.110 * \\
*\end{array}$ \\
\hline Medium-dose polydatin group & 80 & & $\begin{array}{l}1.814 \pm 0.089 * \\
*\end{array}$ & $2.524 \pm 0.104 *$ & $\begin{array}{l}1.084 \pm 0.040 * \\
*\end{array}$ & $1.004 \pm 0.111^{*}$ \\
\hline Low-dose polydatin group & 40 & & $1.831 \pm 0.089 *$ & $2.608 \pm 0.118$ & $1.298 \pm 0.108 *$ & $0.968 \pm 0.114$ \\
\hline
\end{tabular}

Note: Compared with the model group, $* \mathrm{P}<0.05, * * \mathrm{P}<0.01$.

\section{Influence of polydatin on the TNF- $\alpha$, NO and iNOS in the serum of rat}

Compared with the blank control group, the TNF- $\alpha$ level in the serum of rats in model group rises significantly ( $\mathrm{P}<0.01$ ). Compared with the model control group, the high-dose and medium-dose polydatin groups can significantly lower the serum TNF- $\alpha$ level $(\mathrm{P}<0.05)$; besides, the functions of all doses are dose-dependent to some degree. Compared with the normal control group, the NO content and iNOS activity in the serum of rats in the model group rise significantly $(\mathrm{P}<0.01)$. compared with the model group, the NO content and iNOS activity in the serum of rats in the high-dose and medium-dose polydatin groups decrease significantly and there is a significant difference $(\mathrm{P}<0.05)$. The result is shown in Table 3. 
Table 3. Influence of polydatin on TNF- $\alpha$, NO and iNOS expressions in the serum of rat with hyperlipemia $\left(\mathrm{n}=10, \quad \bar{x}_{ \pm s}\right)$

\begin{tabular}{|c|c|c|c|c|}
\hline Group & $\begin{array}{c}\text { Dose } \\
(\mathrm{mg} \cdot \mathrm{kg}-1)\end{array}$ & $\begin{array}{l}\text { TNF- a } \\
(\mathrm{ng} \cdot \mathrm{L}-1)\end{array}$ & $\begin{array}{c}\mathrm{NO} \\
(\mathrm{mmol} \cdot \mathrm{mL}-1)\end{array}$ & $\begin{array}{c}\text { iNOS } \\
(\mathrm{U} \cdot \mathrm{mL}-1)\end{array}$ \\
\hline Normal control group & & $\begin{array}{c}60.230 \pm 1.254 * \\
*\end{array}$ & $\begin{array}{c}4.320 \pm 0.471 * \\
*\end{array}$ & $\begin{array}{c}8.680 \pm 0.514 * \\
*\end{array}$ \\
\hline Model group & & $95.620 \pm 4.216$ & $11.400 \pm 0.775$ & $13.400 \pm 3.582$ \\
\hline Simvastatin group & 5 & $\begin{array}{c}76.660 \pm 4.021 * \\
*\end{array}$ & $\begin{array}{c}5.070 \pm 0.554 * \\
*\end{array}$ & $\begin{array}{c}8.200 \pm 1.770 * \\
*\end{array}$ \\
\hline High-dose polydatin group & 160 & $\begin{array}{c}82.820 \pm 4.578 * \\
*\end{array}$ & $\begin{array}{c}6.830 \pm 1.287 * \\
*\end{array}$ & $\begin{array}{c}8.310 \pm 0.649 * \\
*\end{array}$ \\
\hline Medium-dose polydatin group & 80 & $91.090 \pm 3.813^{*}$ & $\begin{array}{c}9.420 \pm 0.928 * \\
*\end{array}$ & $9.850 \pm 1.613 *$ \\
\hline Low-dose polydatin group & 40 & $93.578 \pm 4.067$ & $\begin{array}{c}10.130 \pm 1.177 \\
*\end{array}$ & $11.140 \pm 1.386$ \\
\hline
\end{tabular}

Note: Compared with the model group, ${ }^{*} \mathrm{P}<0.05$, $* * \mathrm{P}<0.01$

\section{Discussion}

In the process of AS, lipid metabolism and AS has a very close relationship ${ }^{[1]}$. Both LDL and HDL are regarded as risk factors to form AS. At present, the AS is prevented and treated by lowering the LDL level and raising the HDL level; besides, inhabiting the lipid oxidation modification with the antioxidant has become a brand-new therapeutic approach. This paper detected the LDL, HDL, TNF- $\alpha$, NO and iNOS in the observation group and control group respectively. The experimental result shows that high-dose and medium-dose polydatin can significantly lower the LDL, HDL, TNF- $\alpha$, NO and iNOS levels in serum; it indicates that polydatin can give play to its therapeutic action on AS of experimental rats by balancing the blood lipid metabolism, intervening in the inflammatory response and adjusting the generation of NO.

\section{Acknowledgments}

This paper is a funding project by Hubei Department of Education; project No.: 13C871.

\section{References}

[1] Chen Jianguang, Liu Fengtao and Pan Shuzhou et al. Study on the Relationship between AS and Cardiovascular Risk Factors of Patients with Primary Hypertension. Journal of Chinese Practical Diagnosis and Therapy, 2011, 25(9): 924-926.

[2] Cersosimo E, De Fronzora RA. Insulin resistance and endothelial dysfunction: the road map to cardiovascular diseases. Diabetes Metab Res Rev, 2006, 22(6):423-436.

[3] Chen Qiling, Wang Jihua and Ma Jishun et al. influence of Homocysteine on Relationship between Blood Lipid and Carotid Plaque of Patients with Hypertensive Cerebral Apoplexy. Chinese Journal of Arteriosclerosis, 2002, 10 ( 4): 345-346.

[4] RUBBO H, O'DONNELL V. Nitric oxide, peroxynitrite and lipoxygenase in atherogenesis: mechanistic insights. Toxicology, 2005, 208( 2):305-317. 